\title{
Effects of the melatonin on the kidney of high fat diet fed obese rats: A stereological and histological approach
}

\author{
Kıymet Kübra Yurt, Elif Kayhan, B.Zuhal Altunkaynak*, Gamze Tümentemur, Süleyman Kaplan
}

${ }^{a}$ Department of Histology and Embryology, Ondokuz Mayls University, Samsun, Turkey

\section{ARTICLE INFO}

\section{Article History}

Received 25/02/2012

Accepted 22/05/2013

\section{* Correspondence to:}

Berrin Zuhal Altunkaynak

Department of Histology and Embryology,

Faculty of Medicine,

Ondokuz Mayis University, Samsun, Turkey

e-mail: berrinzuhal@gmail.com

\section{Keywords:}

Obesity

Kidney

Melatonin

Glomerulus

Stereology

Physical disector

\section{ABSTRACT}

Obesity is associated with multiple conditions that are known to compromise renal function, including hypertension, diabetes, hyperuricemia, and the metabolic syndrome that can independently have a detrimental effect on renal function. The aim of this study was to examine effects of fatty diet induced obesity and melatonin on kidney in female rats by histological and quantative methods. For this aim, 24 rats were randomly divided into 4 groups including Non-obese control (NC group), Obese control (OC group), Nonobese Melatonin (NOM group) and Obese-Melatonin (OM group) groups. In the obese groups, rats, were fed with high fat diet ( $40 \%$ of calories from fat) for 15 weeks. The rats of the non-obese groups were fed with standard, commercial rat diet during the same period. At the end of the $15^{\text {th }}$ week, rats in the melatonin groups started to received daily injections (i.p.) $10 \mathrm{mg} / \mathrm{kg}$ melatonin for 6 weeks. At the end of the experiment, a serial sections of kidneys were stained with Hematoxylen-eosin. Glomerular number $(\mathrm{Ng})$ is considered a major determinant of renal function and outcome. Unbiased stereological methods have been used to estimate of $\mathrm{Ng}$. Also histopathological analysis was made on the same sections. Stereological examination of the kidneys showed differences in terms of total kidney volume, volume of cortex, medulla and numerical density of glomeruli among the groups. Light microscopic investigation showed a dilatation in blood vessels and Bowman's space, mononuclear cell infiltration, degeneration in nephrons, including glomerulosclerosis and tubular defects, and an increase in the connective tissue in the kidneys in the OC group. But, both stereological deficits and histological damages detected in OC group recovered in OM group after melatonin treatment. We suggested that a fatty diet is responsible for the rats' obesity and may lead to renal deformities as a result of histopathological changes such as vessel dilatation, tubular defects, inflammation and connective tissue enlargement of the kidney. Also melatonin treatment after obesity may contribute structural and functional healing.

J. Exp. Clin. Med., 2013; 30:153-158

(C) 2013 OMU

\section{Introduction}

Obesity is an important chronic disease. It occurs due to more body fat accumulation than normal levels. The average body mass index (BMI) among the patients initiating chronic dialysis increased from $25.7 \mathrm{~kg} / \mathrm{m}^{2}$ in 1995 to $27.5 \mathrm{~kg} / \mathrm{m}^{2}$ in 2002 (Kramer et al., 2006). It is associated with many factors and needs medical treatment (Altunkaynak and Özbek, 2006). Obesity has become a major global health concern, and its incidence has increased sharply in recent years. Obesity is one of the important criteria of the metabolic syndrome that is characterized by the concurrent existence of obesity, dyslipidemia, hyperglycemia, hyperinsulinemia, and hypertension.
It has been shown that obesity or metabolic syndrome is a strong and independent risk factor for cardiovascular disease that causes mortality (Isomaa et al., 2001; Lakka et al., 2002) and for the development of microalbuminuria and end-stage renal disease (Chen et al., 2004). Also, obesity should be considered the most important risk factor for chronic kidney disease (CKD) because of its strong association with diabetes and hypertension, the two most common etiologies of endstage kidney disease (ESKD).

Melatonin ( $\mathrm{N}$-acetyl-5-methoxytryptamine) is synthesized and secreted by pineal gland. This syntesis and secretion has a cyclic rhytm that shows diurnal variation which in- 
creases in the dark phase of the day and decreases in the light phaze of the day (Nowak et al., 1998). It has been reported that melatonine has very close direct and indirect correlation with kidney functions. Renal cortex has receptors for melatonin that is thought to be responsible for glomeruler filtration and renin secretion (Song et al., 1993; Drew et al., 1998). In addition, it was focused on the central hypotensive and endogenous effect of melatonin (Laflamme et al., 1998).

Daily administration of melatonin suppresses abdominal fat and plasma leptin levels of middle-age male rats (Rasmussen et al., 1999). Furthermore, melatonin has regulatory effects of body weight in a high fat-induced obese rat model and may prevent some of the side effects on glucose homeostasis such as decreased insulin sensitivity (Prunet-Marcassus et al., 2003).

Given the critical role of oxidative stress and inflammation in the progression of renal disease, the potent antioxidant/ anti-inflammatory actions of melatonin, and its demonstrated deficiency in CKD, we hypothesized that long-term administration of this agent may retard deterioration of renal function and structure in rats with renal mass reduction (Quiroz et al., 2007). Stereology is a method that utilizes random, systematic sampling to provide unbiased quantitative data. It is an important and efficient tool in many applications of microscopy for estimating number, volume and volume ratios of biological objects.

The aim of this study was to examine histological and morphometrical effects of fatty diet induced obesity and melatonin application on kidney of female rats, and to evaluate possible quantitative changes by unbiased stereological methods.

\section{Metarial and methods}

Twenty-four adult female Wistar Albino rats (180-210 gr each) obtained from the Experimental Research and Application Center of Ondokuz Mayis University were randomly allocated to four groups. The rats were housed in plastic cages (2 animals per cage), maintained under standardized conditions of light (12-h light/dark cycle) and room temperature $\left(22 \pm 2^{\circ} \mathrm{C}\right)$, with free access to food and tap water. The diet consumption of all animals was monitored daily, and the animals were weighed daily.

\section{The procedure of the experiment}

The 24 rats used in this study were randomly divided into 4 groups including Non-obese control (NC group), Obese control (OC group), Non-obese Melatonin (NOM group) and Obese-Melatonin (OM group) groups. In obese groups rats fed with high fat diet ( $40 \%$ of calories from fat) of 21 were weeks. At the end of $15^{\text {th }}$ week; whether the subjects became obese or not was tested by their BMI values (Altunkaynak and Özbek, 2006). Rats of the non-obese groups were fed with standard, commercial rat diet for 21 weeks period. At the end of the $15^{\text {th }}$ week of the feeding period, rats in the melatonin groups started to received $10 \mathrm{ug} / \mathrm{kg}$ (i.p) melatonin treatment for 6 weeks by utilizing the own diets.

\section{Preparation of melatonin solution}

Firstly, Melatonin (Sigma M-5250) was dissolved in 2\% ethanol and then it was secondarily dissolved in sterile deionized water. Daily doses were put in separate tubes and the solu- tions were stored at $-20^{\circ} \mathrm{C}$. The daily dose is removed from the freezer and thawed at room temperature. On the day of injection of $10 \mathrm{mg} / \mathrm{kg}$ melatonin were made intraperitoneally for 42 days.

\section{Histological procedure}

The animals were anesthetized and their BMI was calculated. Subsequently, the anesthetized animals were sacrificed by intra-cardiac perfusion of $10 \%$ formalin solution. Then, whole kidneys were removed from the rats and processed for stereological and light microscopical analyses. Subsequently, kidneys were fixed in $10 \%$ formalin solution and dehydrated in graded alcohol series, embedded in paraffin wax, and serially sectioned using microtome (Leica ${ }^{\circledR}$; Germany). Sections selected according to unbiased sampling interval were stained with hematoxylin-eosin.

\section{Stereological methods}

The volume of kidney (KV) was estimated using the Cavalieri principle. Point counting grids were used to estimate the volumes of cortex and medulla. The total number of glomeruli was calculated using the physical dissector method. A point counting test grid was used for the estimation of sectioned area of kidneys (Figure 1a, b). The point density of the point counting grid was designed to obtain an appropriate coefficient of error (CE) for images of the serial sections. Coefficient of error and coefficient of variation (CV) were estimated according to Gundersen and Jensen' formula (Gundersen and Jensen, 1987). The test grid with systematic array of points is randomly placed on screen of PC. The volumes of the kidneys were estimated with the following formula:

\section{Volume $=\mathbf{t} \times \mathbf{a} / \mathbf{p} \times \Sigma \mathrm{P}$}

Where, " $\mathrm{t}$ " is the section thickness; " $\mathrm{a} / \mathrm{p}$ " is the representing area of each point on the point counting grid and " $\Sigma \mathrm{P}$ " is the total number of the points hitting the sections surface areas.

\section{Estimation of glomerular number}

To estimate the total number of glomeruli in a kidney, glomeruli were, counted using physical disectors (Sterio, 1984) in a known fraction of the kidney. Twenty serial light microscographs were used as disector pairs for estimating, number of glomeruli in each group. Glomeruli seen in the reference section but not in the look-up section were counted (Kaplan et. al., 2005). An unbiased counting frame was placed on the reference and the look-up sections on the screen of the PC to perform the counting according to the disector counting method (Unal et al., 2004).

The bottom and the left hand edges of the counting frame are considered to be the (exclusion) lines together with the extension lines. Other boundaries of the frame and the topright corner were considered to be inclusion points and any particle that hit these lines or was located inside the frame was counted as a dissector particle (Fig. 1C, D). Histopathological examinations were carried out on the images of the same sections at light microscopical level.

Stereoinvestigator: The stereological data was estimated with the physical fractionator and Cavalieri method, using a workstation made with a microscope (Olympus BH-40, Tokyo, Japan) equipped with a matching condenser, a microca- 
tor (LEP Electronic Products Ltd, Hawthorne, NY, USA) to control the movements in z-axis (accuracy $0.5 \mu \mathrm{m}$ ), a motorized stage for stepwise displacement in $\mathrm{x}-\mathrm{y}$ axis (accuracy $1 \mu \mathrm{m}$ ), and a CCD video camera (Sony, Tokyo, Japan) connected to a 17 inches. PC monitor (Philips). The whole system was controlled by the software stereoinvestigator (MicroBrightField Inc., Wiliston,Vt., USA; version 9.0) (Vamecq et al., 1993; Aguila et al., 2003, Gilat et al., 2003; Şahin et al., 2003; Nicol et al., 2005).

\section{Statistical analyses}

Microsoft ${ }^{\circledR}$ SPSS Version 15.0 for Windows was used for statistical analyses. One-way Anova with Bonferroni Post Hoc Test was applied to compare volumes of the kidney and mean numerical density of glomeruli.

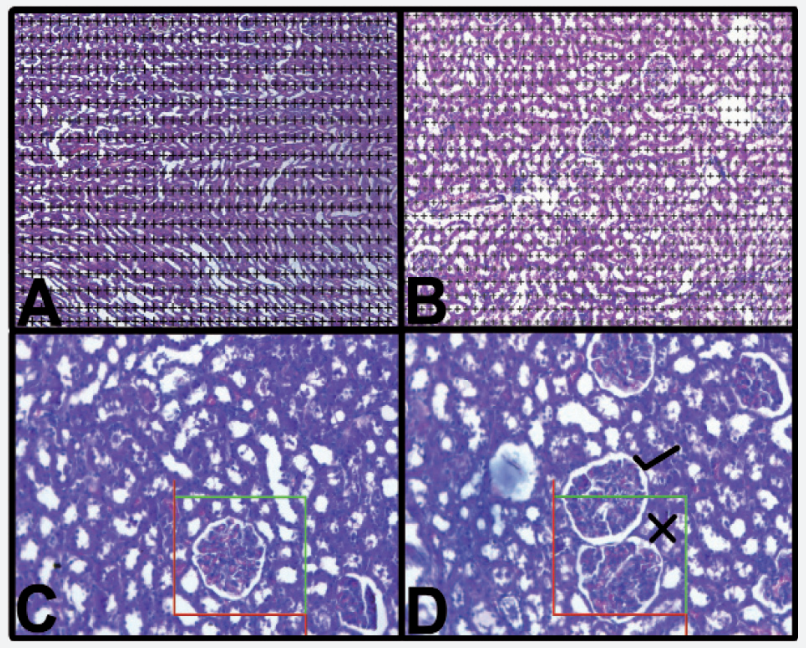

Fig. 1. A, B, Stereological procedures for applying the Cavalieri principle; C, D, Disector pairs which are used for applying the physical dissector. $\checkmark$, show estimated glomeruli hitting the inclusion lines or located inside the frame in both the reference and the look-up sections. $\mathbf{X}$, show non-estimated glomeruli hitting the exclusion lines

\section{Results}

\section{Volumetric results}

\section{Volume of cortex and medulla}

Volumes of cortex and medulla of kidney tissue were additionally estimated by the Cavalieri principle on a series of $\mathrm{H}$-E-stained sections. The estimates of the volumes of the kidney cortex and medulla of control rats and those of obese and melatonin treated rats are given in Figure 2.

The estimation on volumes of cortex and medulla were made with $2.5 \times 2.5 \mathrm{~mm}^{2}$ grid (Fig. 1A, B).

The volumes of the kidney cortex and medulla were 1.30 and $0.82 \mathrm{~cm}^{3}$, respectively, for rats in the control group. By the 15th week of diet application, the volumes of the kidney cortex and medulla were estimated as to be 1.30 and 0.71 $\mathrm{cm}^{3}$, respectively. No significant difference was not seen among the groups by Cavalieri principle in terms of volumetric parameters $(\mathrm{P}>0.05)$ (Fig. 2).

\section{Mean numerical density of Glomeruli (Nv(GI))}

The mean numerical densities of the glomeruli for both groups are shown in Figure 3. There were 246.248 glomeruli/ $\mathrm{cm}^{3}$ in the kidney of the control rats. However, the numeri- cal density of glomeruli in the kidney decreased significantly from the beginning to the end of the experiment. There were approximately 253.070 glomeruli $/ \mathrm{cm}^{3}$ in the kidney of the obese rats Itt was significantly reduced in obese rats $(\mathrm{P}<0.05)$. Specifically, the mean density of glomeruli in the obese group was decreased by $20.3 \%$ relative to the CD group. Numerical density of glomeruli was approximately 273.580 glomeruli $/ \mathrm{cm}^{3}$ in the kidney of the melatonin applied rats. After melatonin treatment, the number of glomeruli significantly increased $(\mathrm{P}<0.05)$ ( Fig. 3).

\section{Histological results}

Glomeruli, vessels, tubules and interstitial structures were normal in the NOC and NOM groups (Fig. 4A, B). When observing these renal specimens by light microscopy on an $\mathrm{H}$-E-stained section, the following glomerular features were seen: The overall cellularity of the glomerulus, the symmetry of the glomerulus and the thickness of the capillary walls (Fig. $4 \mathrm{~A}, \mathrm{~B})$.

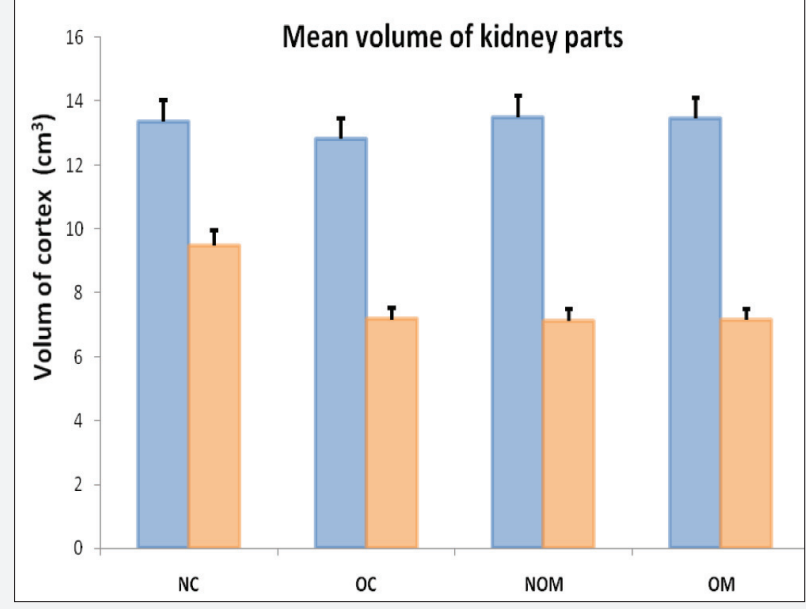

Fig. 2. Volumes of cortex and medulla of kidneys estimated by cavalieri principle of the all groups. There was no significant difference among groups in terms of volumetric data $(\mathrm{P}>0.05)$.

Light microscopic findings of kidney sections from obese rats are summarized as follows. Dilatation in glomerular capillaries and other blood vessels was detected (Fig. 2C). There was eosinophilic cytoplasm in distal and proximal tubules in the cortex. The hydropic degeneration in distal and proximal tubules was widely observed (Fig. 4C and 5C).

The lumens of the tubules and Bowman's space were enlarged and mononuclear cell infiltration was seen in the renal cortices of the obese rats (Fig. 5B, C). Degeneration in nephrons, including glomerulosclerosis and segmental necrosis (Fig. 5B, C) was found. There was cellular debris in the cortex of the kidneys removed from the Obese Control group (OC) (Fig. 5C). After melatonin application no pathological view was detected in light microscopical section of OM group (Fig. 4D). Histological structure of kidneys of the rats in the OM group was smilar to those of the healthy control ones in the NOC group (Fig. 4D).

\section{Discussion}

Obesity disproportionately affects non-white racial/ethnic 
groups and groups with low socioeconomic status. These groups are also those with the highest CKD risk (Shoham et al., 2005).

Krikken et al. examined the potential independent effects of BMI on renal hemodynamics. This study showed that, BMI is associated independently with both glomerular filtration rate (GFR) and filtration fraction also sodium intake modifies this association. This theory is supported by the fact that increased GFR and filtration fraction exist in the absence of obesity-associated comorbidities (Bosma RJ., et al, 2004).

Obesity is a state of increased regional blood flow, arterial pressure, and cardiac output. (Hall et al, 2003a; Hall et al, 2003b). Body size itself directly correlates with glomerular diameter but it does not correlate with the extent of glomerulosclerosis (Kasiske and Napier, 1985). Nephron number is set at birth, but as an individual gains weight, single-nephron GFR must increase to keep pace with metabolic demands.

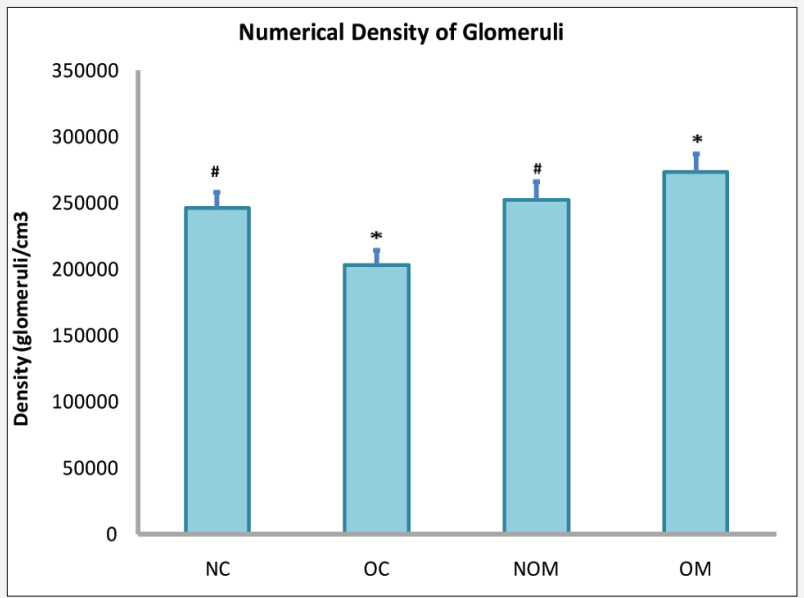

Fig. 3. Numerical densities of glomeruli estimated by physical disector methods of the all groups. There was significant difference between NOC and OC groups (\#) and also between $\mathrm{OC}$ and $\mathrm{OM}$ group $\left({ }^{*}\right)$ in terms of this data $(\mathrm{P}<0.05)$.

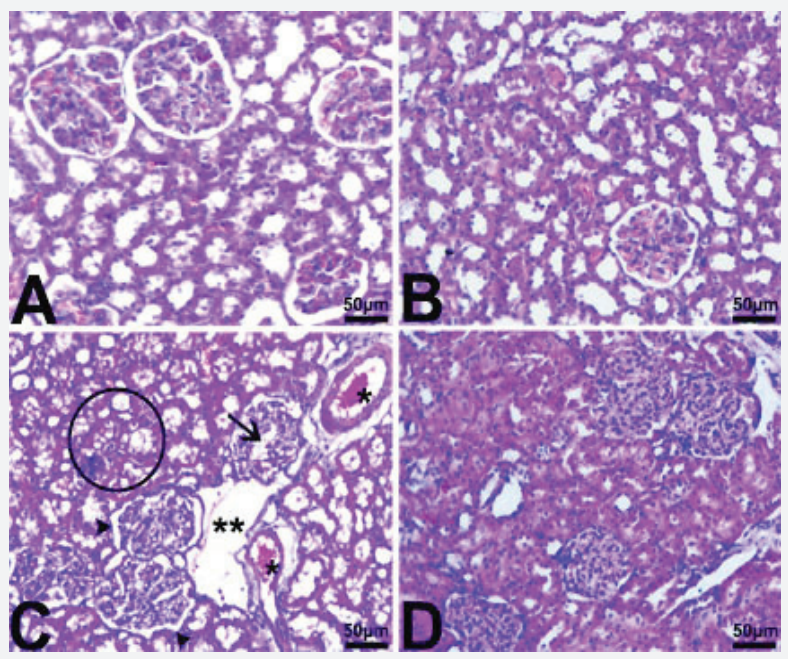

Fig. 4. Light micrographs of kidney tissue from the control group in A, B: Normal kidney. Light micrographs of kidney tissue from the obese group in $\mathbf{C}, \mathbf{D}$. Circle, hydropic degeneration in distal tubules; arrow, glomerular dilatation; *, fibrin accumulation; **, venous dilatation; arrowhead, irregular urinary space. Scale bars $=50 \mu \mathrm{m}$.
Using this logic, those individuals born with the fewest number of nephrons would be expected to hold the highest risk of glomerular hypertrophy given the development of obesity (Griffin et al, 2008).

Melatonin has strong antioxidant effects. Preliminary evidence suggests that it may help strengthen the immune system. Reported the development of hypertension in rats after pinealectomy and the reason for this; increased plasma renin activity and the renin-angiotensin-aldosterone system have been implicated (Burgess et al., 2001; Karppanen et al., 1970; Zanoboni et al., 1967; Girouard et al., 2002).

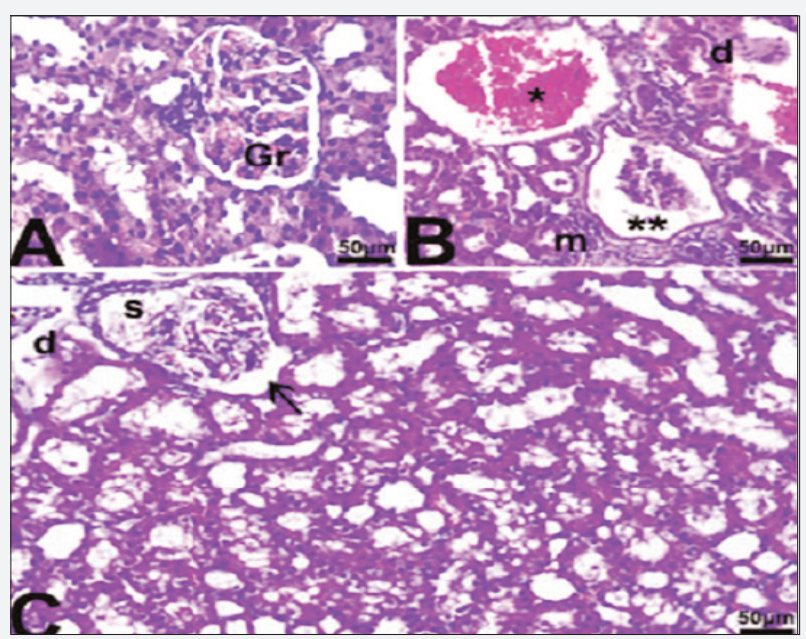

Fig. 5. Light micrographs of kidney tissue in A; Gr, normal glomeruli; d, debris; m, mononuclear cell infiltration; arrow, expanded Bowman space in $\mathbf{B}$; $\mathbf{s}$, segmantal necrosis; hydropic degeneration is seen as wide spread. Scale bars $=50 \mu \mathrm{m}$.

The ability of melatonin to reduce blood pressure, heart rate and sympathetic nerve activity (SNA) is suggesting a hypotensive role with previous reports, exogenous melatonin: (i) can increase the nocturnal drop of diastolic blood pressure in diabetics; (ii) reduce blood pressure in normotensive adults (Ding et al. 2001; Cavallo et al. 2004a); and (iii) significantly reduced nocturnal blood pressure in hypertensive patients (Scheer et al., 2004). These effects suggest the ability of melatonin to modulate both vascular reactivity and catecholamine release (Lusardi et al., 1997; Arangino et al., 1999; Cavallo et al., 2004b). Furthermore, melatonin can act as a hypotensive factor through activation of melatonin receptors in the brain and in the anterior hypothalamic area (Ding et al. 2001).

Obesity is a major health problem that affects up to $30 \%$ of the adult population in developed countries and has been linked to increases in dietary intake, especially fat intake, and a sedentary lifestyle (Kramer \& Luke, 2007). It is well documented that obese patients are associated with serious mortalities, including a high incidence of type 2 diabetes, hyperlipidaemia, hypercholesterolaemia, cardiovascular disease and liver abnormalities, and more attention has been paid to the impact of obesity on renal functions recently (Hall, 1994; (Yilmaz et al. 2002; The following popper user interface control may not be accessible. (Altunkaynak, 2005). Chronic administration of dietetic lipid can cause abdominal obesity 
and can significantly alter the renal cortical structure of rats (Aguila \& Mandarim-De-Lacerda, 2003; the following popper user interface control may not be accessible (Armitage et al. 2005). In the present study, an obesity model was performed by feeding with animal tallow, and the effects of this obesity on rat kidneys were studied.

The mean numerical density, total number, and volume of glomeruli and volume of whole kidney, cortex, medulla from all groups were investigated using stereological methods. Stereological quantifications and histopathological examinations were conducted on light microscopy sections. The results from the two approaches confirmed each other.

Results from quantitative methods, which provide numerical density or geometric data, contribute substantial information on activity of that structure.

According to our stereological results, the numerical density of glomerulus and total number of glomeruli in the OC group was significantly decreased in comparison with the CD group; this indicates that the obesity caused glomerular atrophy. A decrease in the mean volume of glomerulus and volume fraction ratio of all glomeruli the renal cortex and to the whole kidney in the obese group was also found.

We found that the numerical density of glomeruli increased in the OM group compared to the OK group.

Quantifications of the volume of the kidney based on only two-dimensional sections and with a two-dimensional approach have suggested that the mean volume of the kidney in terms of both the cortex and the medulla is increased owing to vasodilatation, connective tissue enlargement or inflammation in the obese group as compared with the control group at the light microscopic level.

Our quantitative findings are consistent with previous reports showing that high fat-dependent obesity is associated with many kidney alterations (Handa and Kreiger, 2002; Jiang et al., 2005; Altunkaynak et al., 2008).

In conclusion, the results of the present study suggest that there is a significant relationship between fatty diet intake and structural changes of the kidney such as a decrease in the numerical density of glomeruli, prominent dilatation of the renal vessels, glomerular necrosis and atrophy, and basal membrane thickening. Also our study show that there is expanded Bowman space, mononuclear cell infiltration, fibrin accumulation, irregular urinary space, hydropic degeneration, segmantal necrosis in obese rats fed with high fat diet.

According to our results, melatonin could prevent the deleterous effects of obesity on kidney structure and glomerular morphology.

\section{Acknowledgements}

This work was performed by funds from the Ondokuz Mayis University (PYO.TIP.1904.11.022).

\section{REFERENCES}

Altunkaynak, B.Z., 2005. Effects of high fat diet induced obesity on female rat livers (a histochemical study). Eur. J. Gen. Med. 2, 100-109.

Altunkaynak, B.Z., Özbek, E., 2006. Obezite: Nedenleri ve tedavi seçenekleri. Van Tıp Dergisi. 13, 138-142.

Altunkaynak M. E., Özbek, E., Altunkaynak, B. Z, Can, I., Unal, D., Unal, B., 2008. The effects of high-fat diet on the renal structure and morphometric parametric of kidneys in rats. J Anat. 6, 845-852.

Aguila, M.B., Mandarim-De-Lacerda, C.A., 2003. Effects of chronic high fat diets on renal function and cortical structure in rats. Exp. Toxicol Pathol. 55, 187-195.

Arangino, S., Cagnacci, A., Angiolucci, M., Vacca, A.M., Longu, G., Volpe, A., Melis, G.B., 1999. Effects of melatonin on vascular reactivity, catecholamine levels, and blood pressure in healthy men. Am. J. Cardiol. 83, 1417-1419.

Armitage, J.A., Lakasing, L., Taylor, P.D., Balachandran, A.A., Jensen, R.I., Dekou, V., Ashton, N., Nyengaard, J.R., Poston, L., 2005. Developmental programming of aortic and renal structure in offspring of rats fed fat-rich diets in pregnancy. J. Physiol. 565, 171-184.

Bosma, R.J., van der Heide, J.J., Oosterop, E.J., de Jong, P.E., Navis, G., 2004. Body mass index is associated with altered renal hemodynamics in non-obese healthy subjects. Kidney Int. 65, 259-265.

Bosma, R.J., Krikken, J.A., Homan, J.J., van der Heide, de Jong, P.E., Navis, G., 2006. Obesity and renal hemodynamics. Contrib. Nephrol. 151, pp. 184-202.

Burgess, H.J., Sletten, T., Savic, N., Gilbert, S.S., Dawson, D., 2001. Effects of bright light and melatonin on sleep propensity temperature and cardiac activity at night. J. Appl. Physiol. 91, 1214-1222.

Cavallo, A., Daniels, S.R., Dolan, L.M., Bean, J.A., Khoury, J.C., 2004a. Blood pressure-lowering effect of melatonin in type 1 diabetes. J. Pineal Res. 36, 262-266.

Cavallo, A., Daniels, S.R., Dolan, L.M., Khoury, J.C., Bean, J.A., 2004b. Blood pressure response to melatonin in type 1 diabetes. Pediatr. Diabetes. 5, 26-31.

Chen, J., Muntner, P., Hamm, L.L., Jones, D.W., Batuman, V., Fonseca, V., Whelton, P.K., He, J., 2004. The metabolic syndrome and chronic kidney disease in U.S. adults. Ann. Intern. Med. 140, 167-174.

Ding, C.N., Cao, Y.X., Zhou, L., Zhu, D.N., Shen, Z.Y., Fei, M.Y., Yu, P., 2001. Effects of microinjection of melatonin and its receptor antagonists into anterior hypothalamic area on blood pressure and heart rate in rats. ActaPharmacol. Sin. 22, 997-1002.

Drew, J.E., Williams, L.M., Hannalý, L.T., Barrett, P., Abramovich, D.R., 1998. Melatonin receptors in the human fetal kidney: 2-[1 125] iodomelatonin binding sites cornelated with expression of Mel 1a and Mel 1b receptor genes. J. Endocrinol. 156, 261-267.

Gilat, T., Leikin-Frenkel, A., Goldiner, I., Juhel, C., Lafont, H., Gobbi, D., Konikoff, F.M., 2003. Prevention of diet-induced fatty liver in experimental animals by the oral administration of a fatty acid bile acid conjugate (FABAC). Hepatology; 38, 436-442.

Girouard, H., Chulak, C., Kejossec, M., Lamontahne, D., Champlain, Y., 2002. Vasorelexant effects of the chronic treatment with melatonin on mesenteric artery and aorta of spontaneously hypertansive rats. J. Hypertens. 19, 1369-1377.

Griffin, K.A., Kramer, H., Bidani, A.K., 2008. Adverse renal consequences of obesity. Am. J. Physiol Renal Physiol. 294, 685-696.

Gundersen, H.J.G., Jensen, E.B., 1987. The efficiency of systematic sampling in stereology and its prediction. J. Microsc. 147, $229-263$.

Hall, J.E., Kuo, J.J., da Silva, A.A., de Paula, R.B., Liu, J., Tallam, L., 2003a. Obesity-associated hypertension and kidney disease. Curr. Opin Nephrol. Hypertens. 195-200.

Hall, J.E., Jones, D.W., Kuo, J.J., da Silva, A., Tallam, L.S., Liu, J., 2003b. Impact of the obesity epidemic on hypertension and renal disease. CurrHypertens Rep. 386-392. 
Hall, J.E., 1994. Renal and cardiovascular mechanisms of hypertension in obesity. Hypertension. 23, 381-394.

Handa, K., Kreiger, N., 2002. Diet patterns and the risk of renal cell carcinoma. Public Health Nutr. 5, 757-767.

Isomaa, B., Almgren, P., Tuomi, T., Forsén, B., Lahti, K., Nissén, M., Taskinen, M.R., Groop, L., 2001. Cardiovascular morbidity and mortality associated with the metabolic syndrome. Diabetes Care. 24, 683-689.

Jiang, T., Wang, Z., Proctor, G., Proctor, G., Moskowitz, S., Liebman, S.E., Rogers, T., Lucia, M.S., Li, J., Levi, M., 2005. Diet-induced obesity in $\mathrm{C} 57 \mathrm{BL} / 6 \mathrm{~J}$ mice causes increased renal lipid accumulation and glomerulosclerosis via a sterol regulatory element-binding protein-1cdependent pathway. J. Biol. Chem. 280, 32317-32325.

Kaplan, S., Gokyar, A., Unal, B., Tunç, A.T., Bahadir, A., Aslan, H., 2005. A simple technique for localizing consecutive fields for disector pairs in light microscopy: Application to neuron counting in rabbit spinal cord following spinal cord injury. J. Neurosci Methods. 145, $277-284$.

Karppanen, H., Vapaatalo, H., Lahovaara, S., Paasonen, M.K., 1970. Studies with pinealectomized rats. Pharmacology. 3, $76-84$.

Kasiske B.L., Napier J., 1985. Glomerular sclerosis in patients with massive obesity. Am J Nephrol. 5, 45-50.

Kramer, H.J., Saranathan, A., Luke, A., et al. 2006. Increasing body mass index and obesity in the incident ESRD population. J Am SocNephrol. $17,1453-1459$.

Kramer, H., Luke, A., Obesity and kidney disease: A big dilemma. 2007. Curr. Opin. Nephrol. Hypertens. 16, 237-241.

Laflamme, A.K., WU, L., Faucart, S., Champlain, Y., 1998. Impaired basal sympathetic tone and alfa1 adrenergic responsiveness in association with the hypotensive effect of melatonin in spontaneously hypertensive rats. Am. J. Hypertens. 11, 219-222.

Lakka, H.M., Laaksonen, D.E., Lakka, T.A., Niskanen, L.K., Kumpusalo, E., Tuomilehto, J., Salonen, JT., 2002. The metabolic syndrome and total and cardiovascular disease mortality in middLle-aged men. J. Am. Med. Assoc. 288, 2709-2716.

Nowak, Y.Z., Zawilsha, Y.B., 1998. Melatonin and its physiological and therapeutic properties. Pharm. World. Sci. 20, 18-27.

Nicol, C.J., Adachi, M., Akiyama, T.E., Gonzalez, F.J., 2005. PPARgamma in endothelial cells influences high fat diet-induced hypertension. Am. J. Hypertens. 18: 549-56.

Praga, M., Hernandez, E., Andres, A., Leon, M., Ruilope, M.L., Rodicio LJ., 1995. Effects of body-weight loss and captopril treatment on proteinuria associated with obesity. Nephron. 70, 35-41.

Praga, M., Hernández, E., Morales, E., et al., 2001. Clinical features and long-term outcome of obesity-associated focal segmental glomerulosclerosis. Nephrol. Dial. Transplant. 16, 1790-1798.

Prunet-Marcassus, B., Desbazeille, M., Bros, A., Louche, K., Delagrange, P., Renard, P., et al., 2003. Melatonin reduces body weight gain in Sprague Dawley rats with diet-induced obesity. Endocrinology. 144, 5347-5352.

Quiroz, Y., Ferrebuz, A., Romero, F., Vaziri, N.D., Rodriguez-Iturbe, B., 2008. Melatonin ameliorates oxidative stress, inflammation, proteinuria, and progression of renal damage in rats with renal mass reduction. Am. J. Physiol Renal Physiol. 294, 336-344.

Rasmussen, D.D., Boldt, B.M., Wilkinson, C.W., Yellon, S.M., Matsumoto, A.M., 1999. Daily melatonin administration at middle age suppresses male rat visceral fat, plasma leptin, and plasma insulin to youthful levels. Endocrinology. 140, 1009-1012.

Sahin, B., Emirzeoglu, M., Uzun, A., Incesu, L., Bek, Y., Bilgic, S., Kaplan, S., 2003. Unbiased estimation of the liver volume by the Cavalieri principle using magnetic resonance images. Eur. J. Radiol. 47: 164-170.

Scheer, F.A., Van Montfrans, G.A,. et al. 2004. Daily nighttime melatonin reduces blood pressure in male patients with essential hypertension. Hypertension. 43, 192-197.

Shoham, D.A., Vupputuri, S., Kshirsagar, A.V., 2005. Chronic kidney disease and life course socioeconomic status: A review. Adv. Chronic Kidney Dis. 12, 56-63.

Song, Y., Poon, A.M.S., Lee, P.P.N., Pamg, S.F., 1993. Putative melatonin receptors in the male guinea pig kidney. J. Pineal Res.15, 153-160.

Sterio, D.C., 1984. The unbiased estimation of number and sizes of arbitrary particles using the disector. J. Microsc. 134, $127-136$.

Unal, B., Özbek, M.E., Aydin, M.D., et al., 2004. Effect of haloperidol on the numerical density of neurons and nuclear height in the rat hippocampus: a stereological and histopathological study. Neurosci Res. Commun. 34, 1-9.

Usardi, P., Preti, P., et al., 1997. Effect of bed time melatonin ingestion on blood pressure of normotensive subjects. Blood. Press. Monit. 2, 99103.

Yilmaz, A., Suleyman, H., Umudum, Z., Sahin, Y.N., 2002. The effect of adrenalectomy on leptin levels and some metabolic parameters in rats with diet-induced obesity. Biol. Pharm. Bull. 25, 580-583.

Zanoboni, A., Zanoboni-Muciaccia, W., 1967. Experimental hypertension in pinealectomized rats. Life Sci. 6, 2327-2331. 